\title{
Radiographic characteristics of neuroendocrine liver metastases do not predict clinical outcomes following liver resection
}

\author{
Emily A. Armstrong ${ }^{1}$, Eliza W. Beal ${ }^{2}$, Manisha Shah $^{3}$, Bhavana Konda ${ }^{3}$, Sherif Abdel-Misih ${ }^{2}$, Aslam Ejaz $^{2}$, \\ Mary E. Dillhoff ${ }^{2}$, Timothy M. Pawlik ${ }^{2}$, Jordan M. Cloyd ${ }^{2}$ \\ ${ }^{1}$ The Ohio State University College of Medicine, Columbus, OH, USA; ${ }^{2}$ Department of Surgery, Division of Surgical Oncology, ${ }^{3}$ Department of \\ Internal Medicine, Division of Medical Oncology, The Ohio State University Wexner Medical Center, Columbus, OH, USA \\ Contributions: (I) Conception and design: All authors; (II) Administrative support: None; (III) Provision of study materials or patients: None; \\ (IV) Collection and assembly of data: EA Armstrong, EW Beal; (V) Data analysis and interpretation: EA Armstrong, All authors; (VI) Manuscript \\ writing: All authors; (VII) Final approval of manuscript: All authors. \\ Correspondence to: Jordan M. Cloyd, MD. Assistant Professor, Department of Surgery, Division of Surgical Oncology, The Ohio State University \\ Wexner, 410 W $10^{\text {th }}$ Ave, N907 Doan Hall, Columbus, OH 43210-1267, USA. Email: Jordan.Cloyd@osumc.edu.
}

Background: Previous research has demonstrated that specific radiographic criteria, including the presence of calcifications and the enhancement pattern on computed tomography (CT) imaging, correlates with clinicopathologic features and outcomes of patients with gastroenteropancreatic neuroendocrine tumors (NET). We sought to investigate whether these radiographic characteristics were prognostic among patients with neuroendocrine liver metastases (NELM) undergoing surgical resection.

Methods: The preoperative contrast-enhanced CT scans of all patients who underwent resection of NELM at a single institution between 2000-2015 were retrospectively reviewed. The presence of calcifications was determined on non-contrast phase imaging. Enhancement on the arterial phase scan was categorized as hyperenhancing, hypoenhancing, or mixed. Relevant clinicopathologic characteristics as well as recurrencefree survival (RFS) and overall survival (OS) were compared between groups.

Results: Among 82 patients who underwent resection of NELM, 57 had available data on calcifications while 51 had data available on arterial enhancement patterns. Among all patients, median age was 58 (IQR: 47-63) and the majority were female $(\mathrm{N}=48,59.5 \%)$. The most common primary tumor locations were pancreas $(\mathrm{N}=25,30.5 \%)$ and small bowel $(\mathrm{N}=27,32.9 \%)$. The most commonly performed operations were right hepatectomy $(\mathrm{N}=29,35.4 \%)$, bisegmentectomy $(\mathrm{N}=15,18.3 \%)$, and segmentectomy $(\mathrm{N}=14,17.1 \%)$. Median tumor number was 4 (IQR: 2-9), median Ki-67 was 5\% (IQR: 2-10\%), and median size of the largest liver metastasis was 4.5 (IQR: $2.8-7.7) \mathrm{cm}$. Twelve (21\%) patients had tumor calcifications. Among patients with and without calcifications there were no differences in demographics, clinicopathologic characteristics, RFS $(\mathrm{P}=0.772)$ or OS $(\mathrm{P}=0.095)$. Arterial enhancement was hypoenhancing in $23(45.1 \%)$, hyperenhancing in 10 (19.6\%), and mixed in 18 (35.3\%). Similarly, there were no differences between arterial enhancement groups in demographics, clinicopathologic characteristics, RFS $(\mathrm{P}=0.618)$ or OS $(\mathrm{P}=0.268)$.

Conclusions: Radiographic characteristics on contrast-enhanced CT are not associated with the outcomes of patients undergoing resection of NELM. Future investigations should evaluate the prognostic impact of functional neuroendocrine imaging.

Keywords: Neuroendocrine tumor (NET); carcinoid; hepatectomy; radiomics; prognosis

Submitted Feb 04, 2019. Accepted for publication May 29, 2019.

doi: 10.21037/hbsn.2019.06.02

View this article at: http://dx.doi.org/10.21037/hbsn.2019.06.02 


\section{Introduction}

Neuroendocrine tumors (NET) are a rare and heterogenous group of neoplasia that develop from enterochromaffin cells of the gastrointestinal tract, lungs, and pancreas. The nature of these tumors ranges from a slow-growing phenotype to aggressive, rapidly growing neoplasms. Well-differentiated NETs typically exhibit an indolent disease presentation and overall survival (OS) rates for patients with localized disease at 5 years range between $43 \%$ and $93 \%$, depending on the primary tumor site (1). Liver metastases from welldifferentiated NETs occur frequently and are associated with a worse prognosis, with a 5-year OS rate between $13 \%$ and 54\% (2-4). Fortunately, multiple treatment options for neuroendocrine liver metastases (NELM) are available, including ablation, transarterial therapies, peptide receptor radionuclide therapy (PRRT), and systemic chemotherapy $(5-8)$. Currently, the only potentially curative treatment for NELM is surgical resection, however, the lack of reliable biomarkers or prognostic factors limits the ability to appropriately select patients who are likely to benefit from surgical treatment (9-13).

Previous research has demonstrated that specific radiographic criteria on computed tomography (CT) imaging correlates with the clinicopathologic features and outcomes of patients with gastroenteropancreatic NETs undergoing surgical resection. For example, hypoenhancement of pancreatic neuroendocrine tumors (PNET) was associated with larger size, intermediate grade, and higher rates of lymph node and synchronous liver metastases as well as worse OS following resection compared to patients with hyperenhancing PNETs (14). In another study, the presence of calcifications on preoperative CT correlated with intermediate tumor grade and the presence of lymph node metastasis, which predicted poor patient outcome (15). Nevertheless, whether similar radiographic criteria are associated with the clinical outcomes of patients undergoing resection of NELM has not been previously investigated. Therefore, the goal of this study was to determine if NELM enhancement pattern and/or the presence of tumor calcifications correlates with other clinicopathologic characteristics or long-term survival after hepatic resection.

\section{Methods}

A retrospective review of all patients with NELM who underwent resection between 2000 and 2015 at the Ohio State University Wexner Medical Center and James Cancer
Hospital and Solove Research Institute were included. Patients who did not have preoperative multi-phase CT scans were excluded. This study was approved by the Institutional Review Board of the Ohio State University.

Individual patient data were then abstracted from the electronic medical record. The following patient variables were considered: age, gender, race, health insurance status, American Society of Anesthesia (ASA) class, Eastern Cooperative Oncology Group (ECOG) status, Charlson comorbidity score, patient comorbidities including diabetes mellitus, hypertension, and chronic obstructive pulmonary disease, and body mass index. The following characteristics of the primary tumor were considered: location of the primary, whether the tumor was functional, patient symptom status, tumor size, grade, Ki-67 and whether liver metastases were synchronous. Characteristics of the liver metastases were also considered including tumor Hounsfeld units (HU) on CT in the arterial phase, calcification status on non-contrast CT, type of operation, size of largest metastasis, number of metastases, tumor grade and Ki-67.

All patients underwent multi-phase CT with thinaxial sections of the abdomen and pelvis with dedicated non-contrast, arterial, portal venous, and delayed phasing imaging prior to surgical resection. For the purposes of this study, all preoperative CT were re-reviewed using Digital Imaging and Communications in Medicine (DICOM, Arlington, VA, USA). Tumor attenuation (Figure 1) was measured by placing an oval region of interest (ROI) of $10 \mathrm{~mm}^{2}$ within the tumor. Care was taken to avoid areas of calcifications, treatment effect (if applicable), or adjacent normal vasculature when assessing tumor enhancement. This was performed three times for each representative liver mass, and the mean of these measurements was used to represent the average tumor attenuation. When multiple tumors were present in one patient, the characteristics of the largest tumor were recorded. Normal liver parenchyma attenuation similarly was defined as the average of three $10 \mathrm{~mm}^{2}$ oval ROI within the liver parenchyma. The degree of tumor enhancement on arterial phase imaging was determined by comparison of the average tumor attenuation to the average surrounding hepatic parenchyma attenuation. Enhancement was categorized as hypo-, hyperor mixed enhancement based on this comparison. Mixed enhancement was defined as a mass exhibiting multiple areas of both hyper- and hypoenhancement compared to the surrounding liver parenchyma. In addition, the presence of calcifications was also assessed by evaluating non-contrast phase imaging (Figure 1). For patients with multiple tumors, 
the presence of calcifications in any lesion was included.

The method of hepatic resection was deferred to the individual surgeon. Following surgery, all patients were followed in a multidisciplinary fashion by surgical and medical oncology. Surveillance imaging with CT chest/ abdomen/pelvis and tumor markers were obtained every 6 months. Recurrence was defined as radiographic evidence of recurrent tumor after a complete (R0 or R1) resection; confirmatory biopsies were not required. Recurrence free survival was defined as the time of resection to the time of recurrence or last follow-up in months. OS was defined as the time of resection to the time of death or last follow-up in months.

First, the clinicopathologic characteristics, operative details, and long-term outcomes of patients with NELM that did and did not contain calcifications were compared. Second, the clinicopathologic characteristics, operative details, and long-term outcomes of patients with NELM who demonstrated hyperenhancement, hypoenhancement, or mixed enhancement were compared. Categorical variables were compared using chi-square tests or Fisher's exact tests, as appropriate. Continuous variables were compared using Students $t$-test or Kruskal-Wallis one-way analysis of variance, as appropriate. Kaplan-Meier analysis and log-rank tests were used to compare recurrencefree survival (RFS) and OS among calcification and arterial enhancement groups. Additionally, supplementary univariate Cox regression analysis was performed to analyze the association of clinicopathologic characteristics and operative details with OS among the entire cohort. All analysis was performed in STATA MP 14.2 (StataCorp, College Station, TX, USA). Statistical significance was assessed at 0.05 .

\section{Results}

Eighty-two patients with NELM met inclusion criteria. Complete demographic, clinicopathologic, and operative data for the entire cohort are reported in Table 1. Most patients had pancreatic $(\mathrm{N}=25,30.5 \%)$ or small bowel $(\mathrm{N}=27,32.9 \%)$ primaries, with the majority grade $1(\mathrm{~N}=38$, $69.1 \%$ ), having a median Ki-67 of 3\% (IQR: 2-10\%). Prior treatment history was heterogeneous: 69 patients $(84.1 \%)$ had received prior systemic chemotherapy, $70(85.3 \%)$ had received prior somatostatin analogs, and 23 (28.0\%) had undergone prior transarterial chemoembolization. Twelve (21.1\%) patients had calcifications in their NELM. Arterial enhancement status was hypoenhancing ( $\mathrm{N}=23,45.1 \%)$, hyperenhancing $(\mathrm{N}=10,19.6 \%)$ and mixed $(\mathrm{N}=18,35.3 \%)$. The most commonly performed operations were right hepatectomy ( $\mathrm{N}=29,35.4 \%)$, bisegmentectomy ( $\mathrm{N}=15$, $18.3 \%)$, and anatomic segmentectomy $(\mathrm{N}=14,17.1)$. The median largest tumor size was 4.5 (IQR: $2.8-7.7$ ) $\mathrm{cm}$ and the median tumor number was 4 (IQR: 2-9). Median follow-up after first liver resection in the entire patient cohort was 42 (IQR: 30-79) months. RFS in the entire cohort was 34.5 (IQR: 23-57) months after first liver resection, while median OS after first liver resection was 44 (IQR: 31-80) months. Factors significantly associated with OS on univariate Cox regression analysis among the entire cohort included BMI (HR, 0.86, 95\% CI: 0.76, 0.96, $\mathrm{P}=0.009)$ and differentiation of the liver metastasis (HR, 5.67, 95\% CI: 1.97, 16.26, P=0.001), whereas Ki67 (HR, 2.62, 95\% CI: $0.99,6.97, \mathrm{P}=0.053$ ) did not quite reach statistical significance (Table S1).

\section{Tumor calcification}

Fifty-seven patients (69.5\%) had noncontrast CT images which could be analyzed for presence of calcifications. The clinicopathological and demographic characteristics of this cohort are reported in Table 2 and, in general, were similar to the overall cohort. Of this group, 12 (21.1\%) had calcifications associated with their NELM. Compared to patients without calcifications, patient demographics, including age, gender, race, and insurance status, were not significantly different in the calcification patient group (all $\mathrm{P}>0.05$ ) although there were some differences in ASA class, ECOG performance status, and comorbidities (Table 2).

On univariate analysis, primary tumor location, functionality, size, grade, Ki-67\%, and presence of synchronous liver metastases were not associated with development of liver tumor calcification $(\mathrm{P}>0.05)$. Furthermore, calcifications were not significantly associated with the size of the largest metastasis, number of liver metastases, tumor grade, Ki-67\%, or tumor enhancement patterns demonstrated on CT A/P $(\mathrm{P}>0.05)$. Median RFS after first liver resection was 32 (IQR: 16-49) months in the group without calcifications and 34 (IQR: 32-45) months in the group with calcifications. Median OS after first liver resection was 41 (IQR: 30-70) months in the group without calcifications and 66 (IQR: 30-113) months in the group with calcifications. On Kaplan-Meier survival analysis, there was no difference in RFS (Figure $2 A, \mathrm{P}=0.772$ ) or OS (Figure $2 B, \mathrm{P}=0.095$ ) between patients with NELM with and without calcifications. 
Table 1 Clinicopathologic characteristics of patients with neuroendocrine liver metastasis $(\mathrm{N}=82)$

\begin{tabular}{|c|c|}
\hline Characteristics & $\mathrm{N}(\%)$ \\
\hline Age (years), median [IQR] & 58 [47-63] \\
\hline Gender, female & $48(58.5)$ \\
\hline \multicolumn{2}{|l|}{ Race } \\
\hline White & $75(91.5)$ \\
\hline Black & $6(7.3)$ \\
\hline Other & $1(1.2)$ \\
\hline \multicolumn{2}{|l|}{ Health insurance } \\
\hline Uninsured & $3(4.2)^{+}$ \\
\hline Government & $22(31.0)^{+}$ \\
\hline Private & $46(65.0)^{+}$ \\
\hline \multicolumn{2}{|l|}{ ASA class } \\
\hline ॥ & $8(10.0)^{+}$ \\
\hline III & $68(84.0)^{+}$ \\
\hline IV & $5(6.0)^{+}$ \\
\hline \multicolumn{2}{|l|}{ ECOG performance status } \\
\hline 0 & $24(30.8)^{+}$ \\
\hline 1 & $50(64.1)^{+}$ \\
\hline 2 & $4(5.1)^{+}$ \\
\hline Charlson comorbidity score, median [IQR] & $8[7-8]$ \\
\hline Diabetes & $12(15.2)^{+}$ \\
\hline Hypertension & $33(41.8)^{+}$ \\
\hline Chronic obstructive pulmonary disease & $3(3.8)^{+}$ \\
\hline Body mass index $\left(\mathrm{kg} / \mathrm{m}^{2}\right)$, median [IQR] & $27.8[23.6-31.0]$ \\
\hline \multicolumn{2}{|l|}{ Primary tumor } \\
\hline \multicolumn{2}{|l|}{ Location of primary } \\
\hline Pancreas & $25(30.5)$ \\
\hline Small bowel & $27(32.9)$ \\
\hline Other & $30(36.6)$ \\
\hline Symptomatic & $61(83.6)^{+}$ \\
\hline Tumor size (cm), median [IQR] & $3.8[2.2-5.5]$ \\
\hline Synchronous liver metastasis & $66(81.5)^{+}$ \\
\hline \multicolumn{2}{|l|}{ Tumor grade } \\
\hline Grade 1 & $38(69.1)^{+}$ \\
\hline Grade 2 & $12(21.8)^{+}$ \\
\hline Grade 3 & $5(9.1)^{+}$ \\
\hline Ki-67, median [IQR] & $3 \%[2-10 \%]$ \\
\hline
\end{tabular}

Table 1 (continued)
Table 1 (continued)

\begin{tabular}{|c|c|}
\hline Characteristics & $\mathrm{N}(\%)$ \\
\hline \multicolumn{2}{|l|}{ Liver Metastasis } \\
\hline Calcifications present & $12(21.1)^{+}$ \\
\hline \multicolumn{2}{|l|}{ Arterial enhancement } \\
\hline Hypoenhancing & $23(45.1)^{+}$ \\
\hline Hyperenhancing & $10(19.6)^{+}$ \\
\hline Mixed enhancement & $18(35.3)^{+}$ \\
\hline \multicolumn{2}{|l|}{ Type of operation } \\
\hline Right hepatectomy & $29(35.4)$ \\
\hline Anatomic bisegmentectomy & $15(18.3)$ \\
\hline Anatomic segmentectomy & $14(17.1)$ \\
\hline Other & $24(29.3)$ \\
\hline Size of largest metastasis (cm), median [IQR] & $4.5[2.8-7.7]$ \\
\hline Tumor number, median [IQR] & $4[2-9]$ \\
\hline \multicolumn{2}{|l|}{ Tumor grade } \\
\hline Grade 1 & $48(67.6)^{+}$ \\
\hline Grade 2 & $19(26.8)^{+}$ \\
\hline Grade 3 & $4(5.6)^{+}$ \\
\hline Ki-67, median [IQR] & $5 \%[2-10 \%]$ \\
\hline Length of stay (days), median [IQR] & $6[5-7]$ \\
\hline \multicolumn{2}{|c|}{$\begin{array}{l}{ }^{+} \text {, Indicates that percentages have been calculated using th } \\
\text { number of patients with available data. ASA, American Societ } \\
\text { of Anesthesiology; ECOG, Eastern Cooperative Oncolog } \\
\text { Group. }\end{array}$} \\
\hline
\end{tabular}

\section{Tumor arterial enhancement pattern}

Fifty-one (of 82, 62.2\%) patients had triple phase CT A/ $\mathrm{P}$ performed. The clinicopathological and demographic characteristics of this cohort are reported in Table 3 and, in general, were similar to the overall cohort. Of these 51 patients, most had NELM demonstrating hypoenhancement $(\mathrm{N}=23,45.1 \%)$, followed by mixed enhancement $(\mathrm{N}=18,35.3 \%)$, and hyperenhancement ( $\mathrm{N}=10,19.6 \%)$ patterns. Patients who had hypoenhancing, hyperenhancing, and mixed enhancement tumors had a median arterial attenuation of 45.4 (IQR: 32.7-56.1) HU, 87.2 (IQR: 78.5-109.8) HU, and 67.5 (IQR: 57.8-76.8) $\mathrm{HU}$, respectively. Venous phase tumor enhancement for hypoenhancing, hyperenhancing, and mixed enhancement tumors were found to be 64.0 (IQR: 50.0-77.9) HU, 91.6 (IQR: 78.7-107.8) HU, and 83.5 (IQR: 66.5-99.0) HU, 

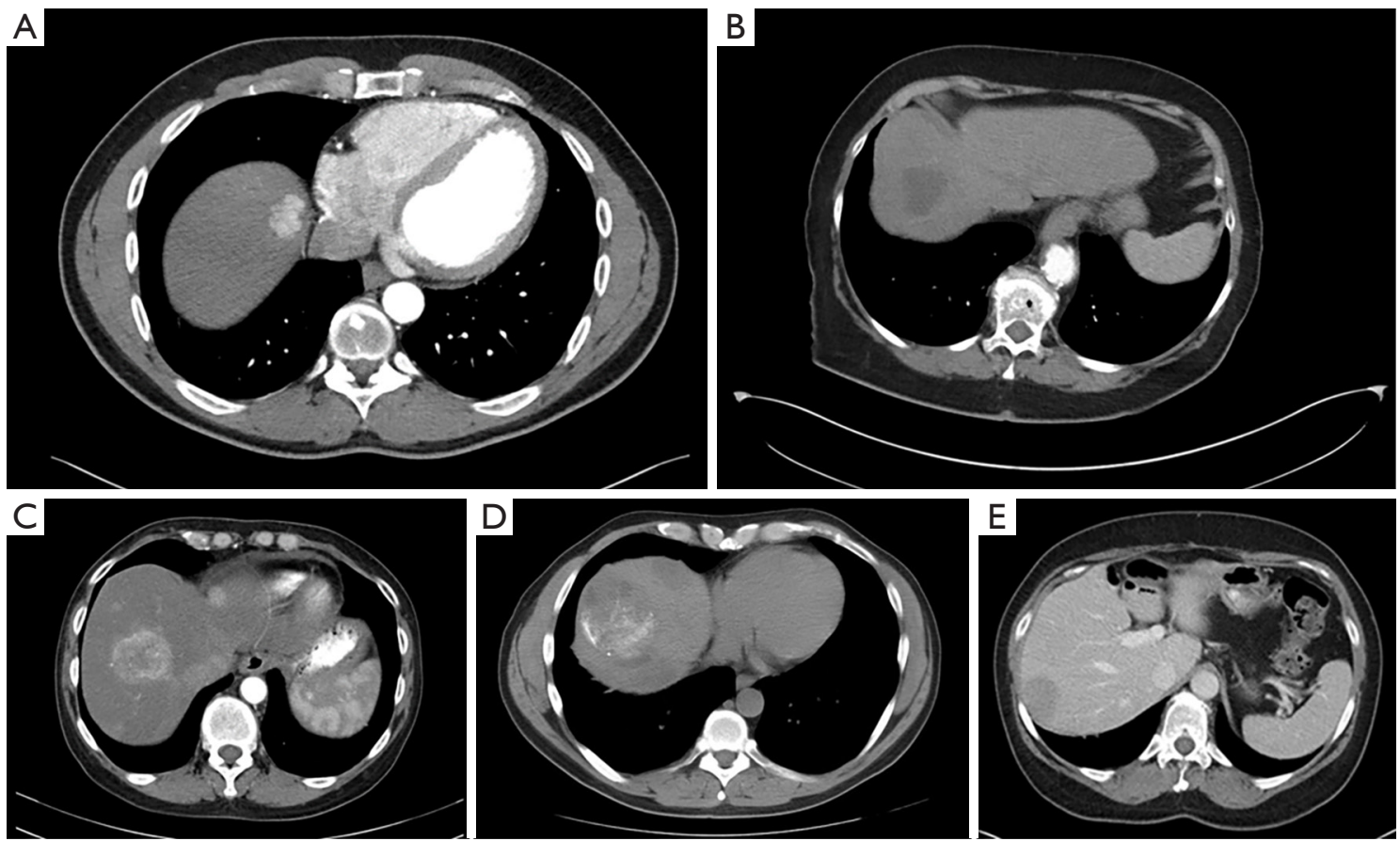

Figure 1 Neuroendocrine liver metastasis. (A) With arterial enhancement; (B) without arterial enhancement; (C) with mixed enhancement; (D) with calcifications; (E) without calcifications.

Table 2 Clinicopathologic characteristics of patients with neuroendocrine liver metastasis by calcification status (N=57)

\begin{tabular}{|c|c|c|c|c|}
\hline Characteristics & $\begin{array}{l}\text { Total cohort } \\
(\mathrm{N}=57), \mathrm{N}(\%)\end{array}$ & $\begin{array}{l}\text { No calcifications present } \\
\qquad(\mathrm{N}=45), \mathrm{N}(\%)\end{array}$ & $\begin{array}{l}\text { Calcifications present } \\
\qquad(\mathrm{N}=12), \mathrm{N}(\%)\end{array}$ & $P$ value \\
\hline Age (years), median [IQR] & 57 [50-63] & 55 [48-60] & 59 [50-63] & 0.286 \\
\hline Race & & & & $0.623^{*}$ \\
\hline White & $53(93.0)$ & $42(93.3)$ & $11(91.7)$ & \\
\hline Health insurance & & & & $0.371^{*}$ \\
\hline Uninsured & $2(3.8)^{+}$ & $1(2.4)^{+}$ & $1(9.1)^{+}$ & \\
\hline Government & $17(32.1)^{+}$ & $13(31.0)^{+}$ & $4(36.4)^{+}$ & \\
\hline III & $49(86.0)$ & 42 (93.3) & $7(58.3)$ & \\
\hline IV & $3(5.2)$ & $1(2.2)$ & $2(16.7)$ & \\
\hline
\end{tabular}

Table 2 (continued) 
Table 2 (continued)

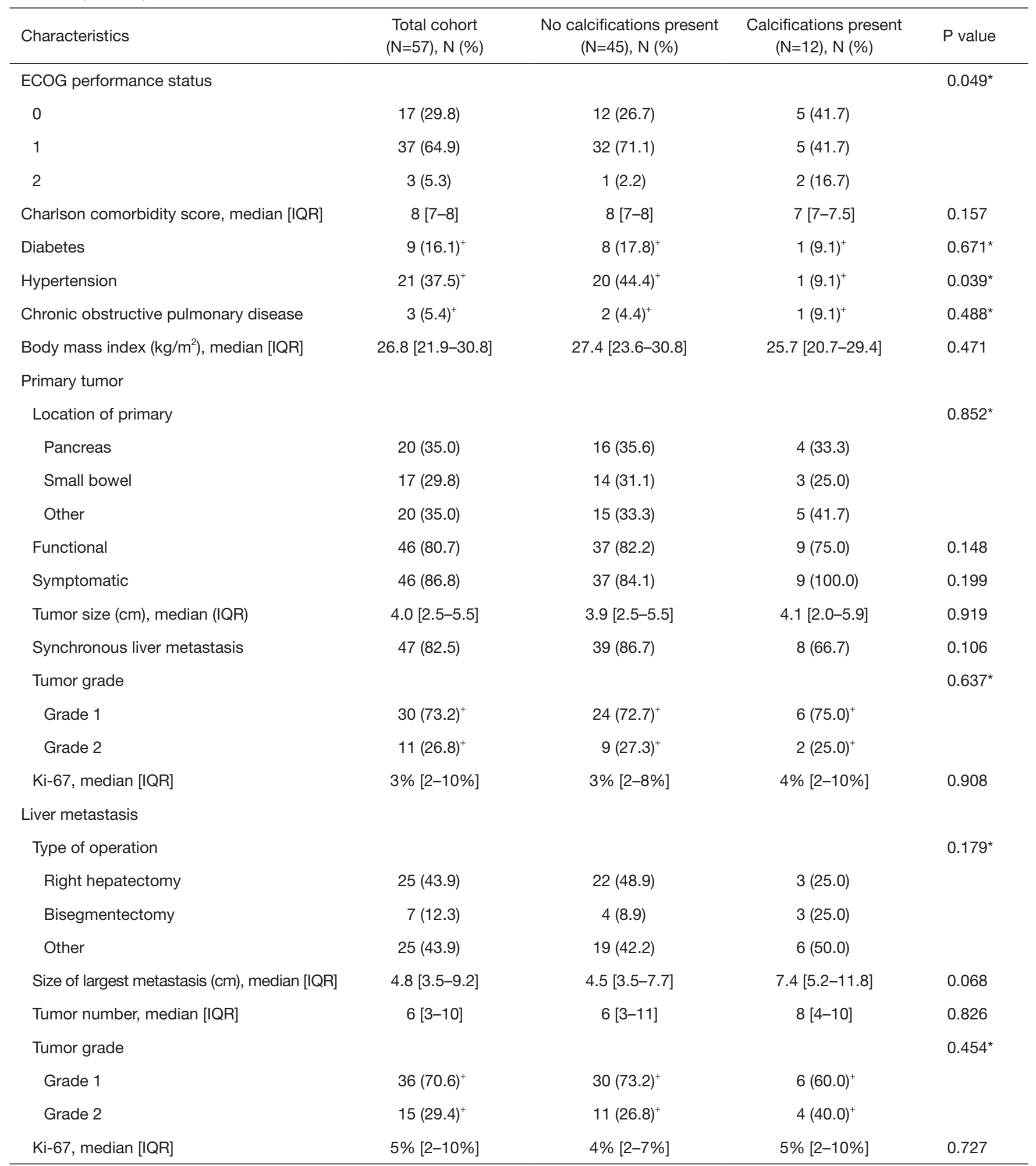

*, Fisher's exact test; ${ }^{+}$, indicates that percentages have been calculated using the number of patients with available data. ASA, American Society of Anesthesiology; ECOG, Eastern Cooperative Oncology Group. 
A

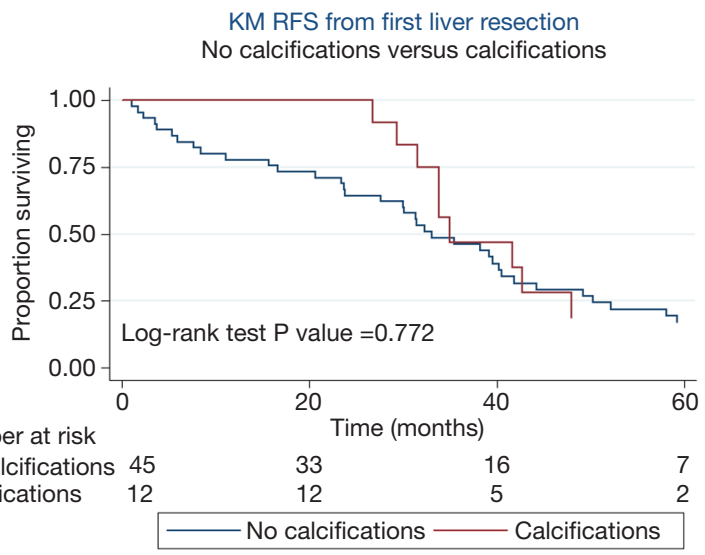

B

KM OS from first liver resection No calcifications versus calcifications

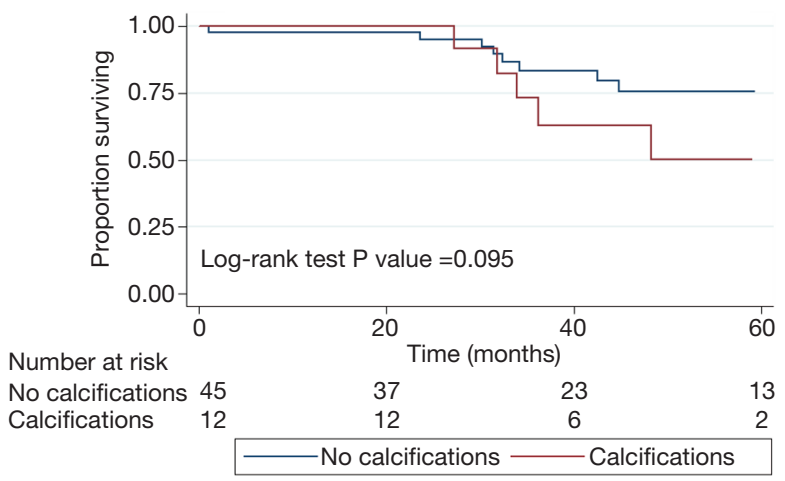

Figure 2 Kaplan-Meier curve demonstrating. (A) Recurrence-free survival (RFS) (log-rank $\mathrm{P}=0.772$ ); and (B) overall survival (OS) (log-rank $\mathrm{P}=0.095$ ) from first liver resection for neuroendocrine liver metastasis comparing calcification groups.

Table 3 Clinicopathologic characteristics of patients with neuroendocrine liver metastasis by arterial enhancement status (N=51)

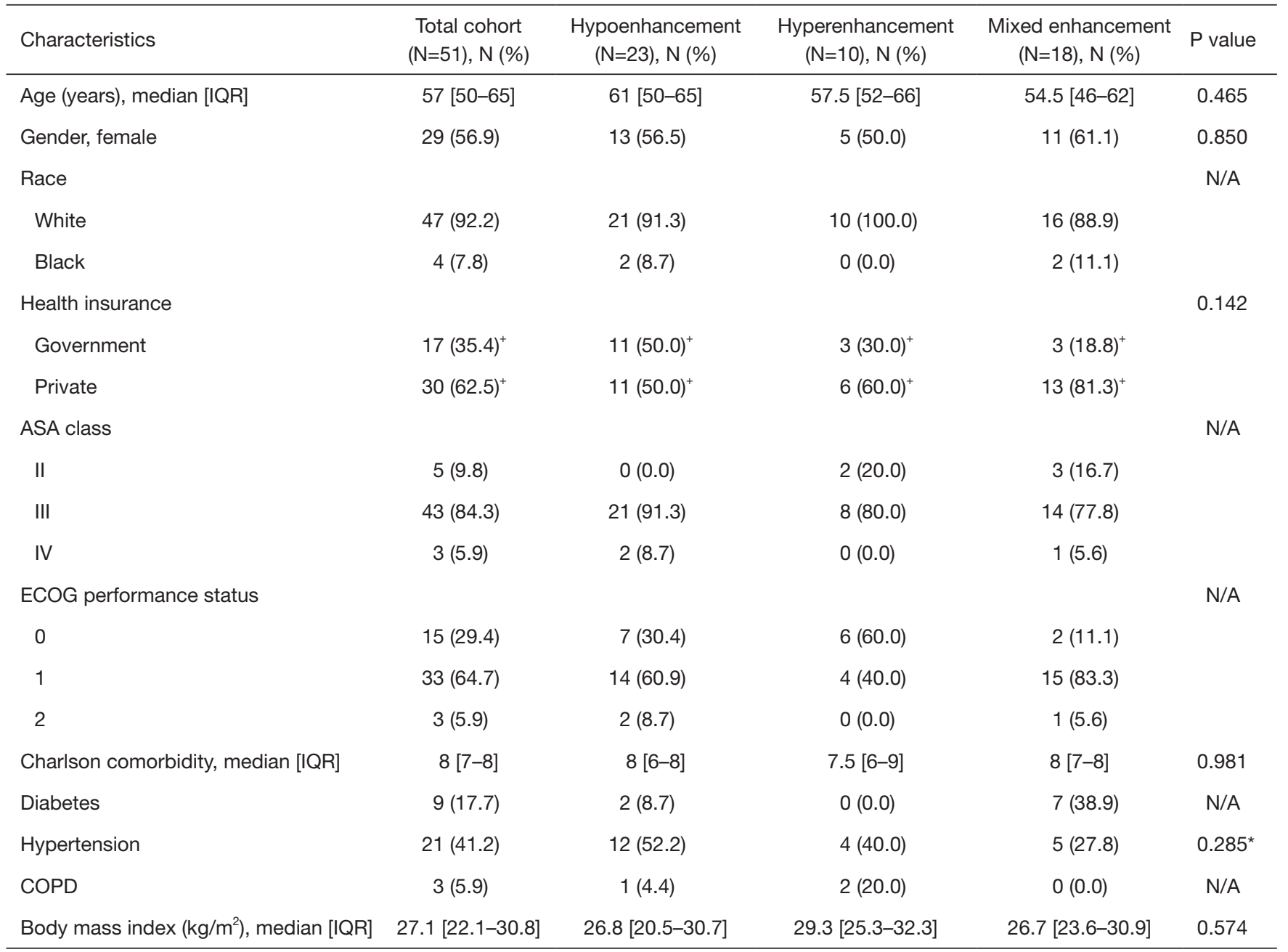

Table 3 (continued) 
Table 3 (continued)

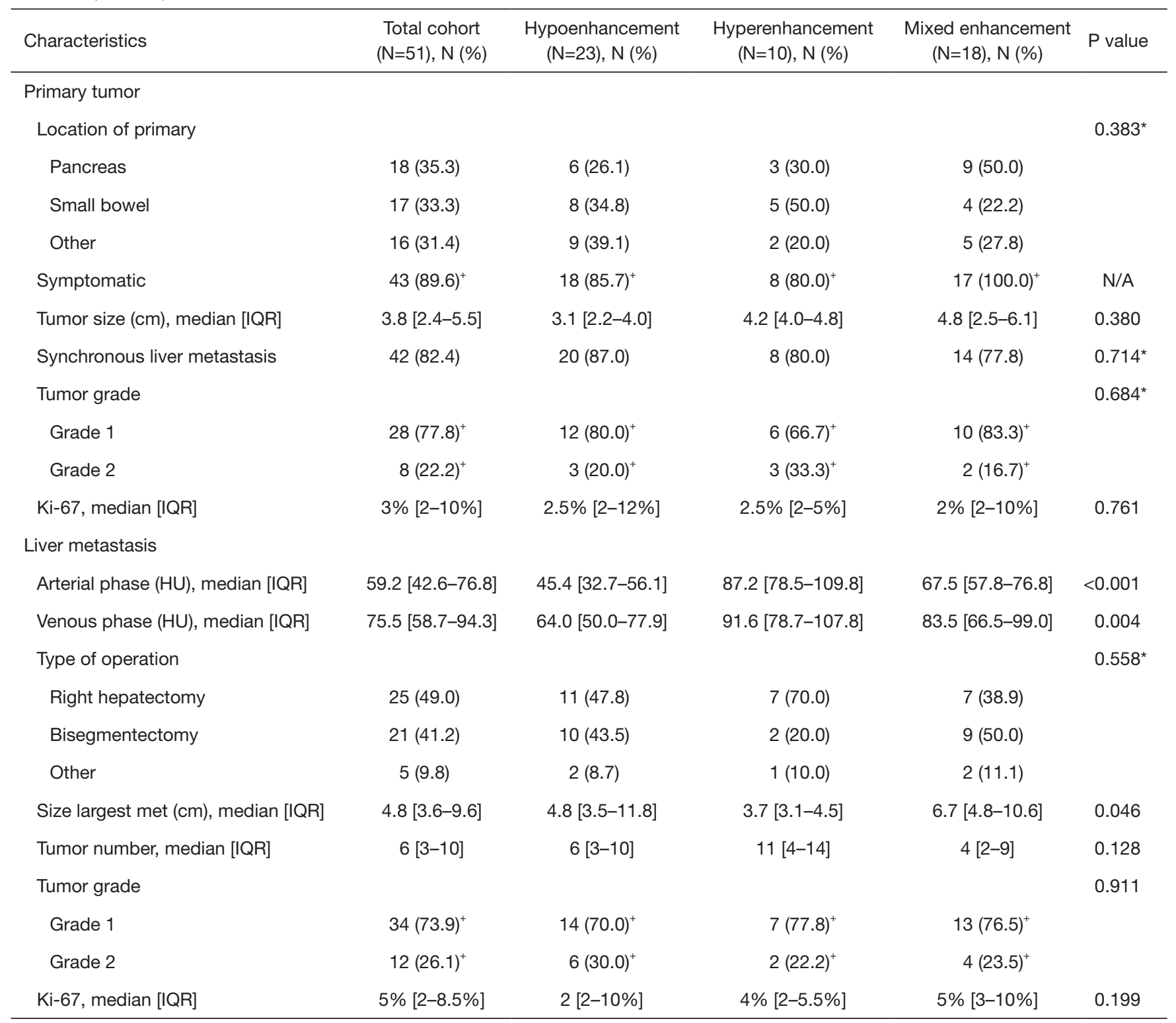

*, Fisher's exact test; ${ }^{+}$, indicates percentages were calculated based on the number of patients with available data. COPD, chronic obstructive pulmonary disease; ASA, American Society of Anesthesiology; ECOG, Eastern Cooperative Oncology Group.

respectively. Demographic and clinicopathological features of patients were not significantly different for any of the three enhancement status patient cohorts $(\mathrm{P}>0.05)$.

On univariate analysis, primary tumor location, functionality, grade, or Ki-67\%, size, symptomatic disease, synchronous disease, or liver metastasis size, number, grade, or Ki-67\% were significantly associated with NELM enhancement status $(\mathrm{P}>0.05)$. Median recurrence free survival after first liver resection was 31 months (IQR: 23-
49) in the hypoenhancing group, 30 (IQR: 15-50) months in the hyperenhancing group and 34 (IQR: 16-63) months in the mixed enhancement group. OS after first liver resection was 45 (IQR: 32-75) months in the hypoenhancing group, 36 (IQR: 16-80) months in the hyperenhancing group and 36 (IQR: 30-63) months in the mixed enhancement group. On Kaplan-Meier survival analysis, there was no significant difference in RFS (Figure $3 A, \mathrm{P}=0.618$ ) or OS (Figure $3 B$, $\mathrm{P}=0.268$ ) among arterial enhancement groups. 
A

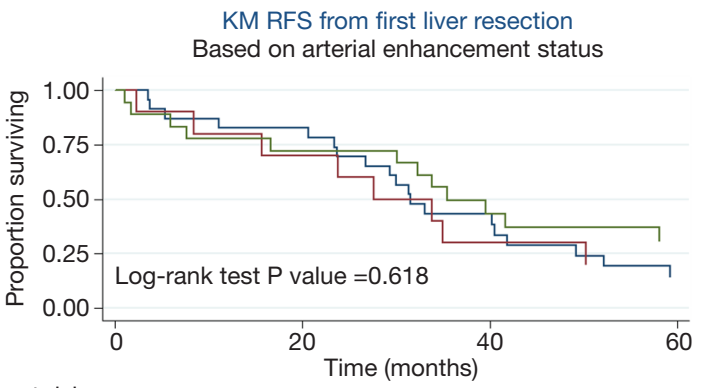

Number at risk

Hypoenhancing 23

Hyperenhancing 10

Mixed

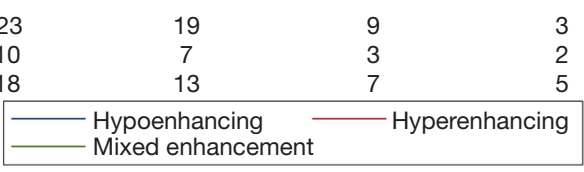

B

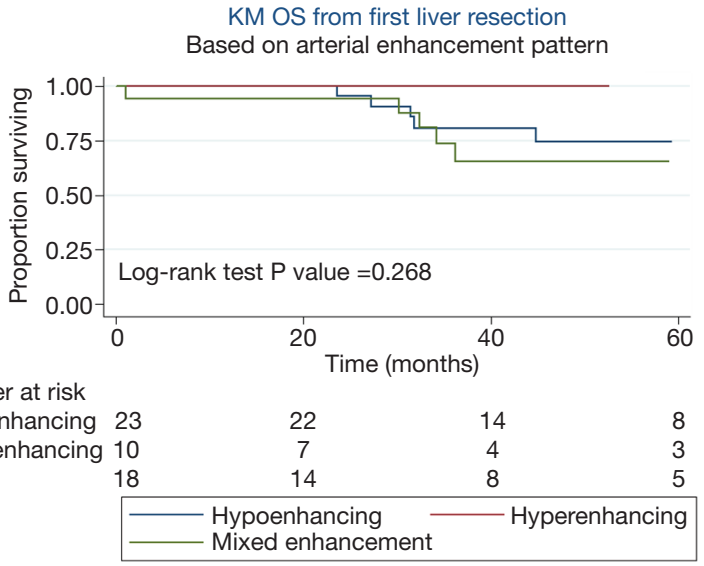

Figure 3 Kaplan-Meier curve demonstrating (A) Recurrence-free survival (RFS) (log-rank P=0.618); and (B) overall survival (OS) (log-rank $\mathrm{P}=0.268$ ) from first liver resection for neuroendocrine liver metastasis comparing arterial enhancement groups.

\section{Discussion}

In this study, we investigated the prognostic significance of radiographic features on the preoperative CT imaging of patients with NELM undergoing surgical resection. Our study had several interesting findings. First, enhancement patterns were quite variable with hypoenhancement representing the most common pattern (45.1\%), followed by mixed-enhancement $(35.3 \%)$ and hyperenhancement (19.6\%). Second, tumor calcifications were relatively common with $21.0 \%$ of patients demonstrating some degree of tumor calcification. Third, neither radiographic characteristic correlated with the outcomes of patients undergoing resection of NELM. These findings suggest that traditional clinicopathologic criteria (e.g., Ki-67 rate, disease free interval, number of tumors) should be used to guide treatment decision making until novel biomarkers are identified that improve patient selection for hepatic resection.

To date, there have been very few studies characterizing NELM CT features. One study of 78 patients with 559 NELMs characterized the enhancement patterns demonstrated by triple-phase CT of NELM of pancreatic primary origin (pNELM) to those of enteric primary origin (eNELM). Their findings demonstrated that a majority of both pNELM and eNELM demonstrated arterial hyperenhancement (including tumors which demonstrated heterogenous enhancement but were predominantly hyperenhancing), indicating that these tumors were hypervascular (16). Another study indicated that hepatic metastases from gastric NET were more likely to show hyperenhancing patterns than hepatic metastases from gastric adenocarcinomas (17). While our study also demonstrated that a majority of the analyzed tumors demonstrated some degree of hypervascularity with $54.8 \%$ of tumors demonstrating purely hyperenhancing or partially hyperenhancing features, their results indicated that $72 \%$ of NELM showed heterogeneous contrast enhancement patterns. This is in contrast to the $35.2 \%$ mixed enhancement pattern in the current study. Additionally, the study indicated that pNELM could potentially be differentiated from eNELM through analysis of non-contrast and portal venous phases of CT A/P, with pNELM more likely demonstrating hyperenhancement on contrast imaging and eNELM more likely demonstrating hypoenhancement on portal venous phase (16). This knowledge could potentially be very useful in cases where the location of the primary tumor is unknown, which is the case in up to $13 \%$ of all NET $(1,18)$.

While these previous studies were important for characterizing the enhancement patterns of various NELM, our study is one of the first to study its prognostic importance. While the enhancement pattern does not appear to correlate with post-resection outcomes, these features are important outside the scope of surgical treatment. Hypervascular NELM demonstrating a hyperenhancing pattern on CT arterial phase is significantly more amenable to liver-directed transarterial therapy than hypoenhancing lesions $(19,20)$.

The importance of calcifications in NELM has been even less discussed in the current literature. With respect to primary NETs, the presence of calcifications in PNETs 
has been shown to correlate with intermediate grade and presence of lymph node metastases (15). Although the frequency of PNET calcifications is uncommon, the presence of focal, centrally-located, coarse calcifications on CT can potentially differentiate PNET from other types of pancreatic tumors which typically show different calcification patterns (21). Ours is the first study to investigate the potential importance of tumor calcifications on NELMs, finding no association with long-term outcomes of patients undergoing resection. It is possible that the biological importance of calcifications becomes less significant when metastases have developed, as compared to in the primary tumor.

Radiomics is an increasingly important field in clinical and translational medicine. Radiographic tumor markers have demonstrated importance in predicting patient prognosis and response to therapy. One study by Kim et al. concluded that pNETs demonstrating uncommon CT findings, including poorly defined borders, heterogeneous enhancement, hypovascularity, and duct dilation correlated with higher tumor grade and poorer survival rates compared to PNETs that did not demonstrate these characteristics (22). Another study suggested radiographic characteristics may be most useful in combination with clinical data in predicting PNET characteristics, such as tumor grade (23). While the results of this study were negative, improvements in functional imaging raise possibilities for novel predictive markers which may not only predict patient prognosis but may also aid in surgical planning (24-26).

There are several limitations to our study, namely the single-institution, retrospective design and relatively small sample size. Several patients in this study received prior therapy and the impact of such therapy on the radiographic characteristics of NELM is unknown. In addition, given the retrospective nature of the study, differences in imaging methodology were possible. For example, factors such as pre-enhancement set point, which has been shown to have subjectivity in observer variation, can affect CT perfusion, leading to variability in determining tumor vascularity (27). On the other hand, radiographic protocols for patients with NETs undergoing CT at our institution are standardized, which should have minimized these biases. Furthermore, all the patients analyzed in our study underwent hepatic resection, so there was significant degree of selection bias within the analyzed patient population. In general, patients who undergo hepatic resection for NELM often have better prognoses than patients who are not surgical candidates $(3,28,29)$. While the goal of this study was to primarily investigate the relationship of CT imaging characteristics and post-surgical resection outcomes, future studies could include non-surgical NELM patients to determine the relationship between CT characteristics with prognostic factors such as tumor grade as well as long-term survival. Given the relatively small sample size, future investigations may be best performed in a multi-institutional fashion.

\section{Conclusions}

In conclusion, radiographic characteristics on contrastenhanced CT, specifically arterial enhancement pattern and presence of calcifications, are not associated with the outcomes of patients undergoing resection of NELM. Future investigations should evaluate the prognostic impact of functional neuroendocrine imaging among a broader patient population.

\section{Acknowledgments}

Funding: The project described was supported by Award Number Grant UL1TR002733 from the National Center for Advancing Translational Sciences.

\section{Footnote}

Conflicts of Interest: The authors have no conflicts of interest to declare.

Ethical Statement: The authors are accountable for all aspects of the work in ensuring that questions related to the accuracy or integrity of any part of the work are appropriately investigated and resolved. The study was approved by the Institutional Review Board of the Ohio State University.

\section{References}

1. Yao JC, Hassan M, Phan A, et al. One hundred years after "carcinoid": epidemiology of and prognostic factors for neuroendocrine tumors in 35,825 cases in the United States. J Clin Oncol 2008;26:3063-72.

2. Riihimäki $M$, Hemminki A, Sundquist K, et al. The epidemiology of metastases in neuroendocrine tumors. Int J Cancer 2016;139:2679-86.

3. Frilling A, Modlin IM, Kidd M, et al. Recommendations for management of patients with neuroendocrine liver metastases. Lancet Oncol 2014;15:e8-21. 
4. Lawrence B, Gustafsson BI, Chan A, et al. The epidemiology of gastroenteropancreatic neuroendocrine tumors. Endocrinol Metab Clin North Am 2011;40:118 , vii.

5. Mayo SC, Herman JM, Cosgrove D, et al. Emerging approaches in the management of patients with neuroendocrine liver metastasis: role of liver-directed and systemic therapies. J Am Coll Surg 2013;216:123-34.

6. Liu CT, Chen MH, Chen JS, et al. The efficacy and safety of everolimus for the treatment of progressive gastroenteropancreatic neuroendocrine tumors: A multiinstitution observational study in Taiwan. Asia Pac J Clin Oncol 2016;12:396-402.

7. Tomozawa Y, Jahangiri Y, Pathak P, et al. LongTerm Toxicity after Transarterial Radioembolization with Yttrium-90 Using Resin Microspheres for Neuroendocrine Tumor Liver Metastases. J Vasc Interv Radiol 2018;29:858-65.

8. Do Minh D, Chapiro J, Gorodetski B, et al. Intra-arterial therapy of neuroendocrine tumour liver metastases: comparing conventional TACE, drug-eluting beads TACE and yttrium-90 radioembolisation as treatment options using a propensity score analysis model. Eur Radiol 2017;27:4995-5005.

9. Fairweather M, Swanson R, Wang J, et al. Management of Neuroendocrine Tumor Liver Metastases: LongTerm Outcomes and Prognostic Factors from a Large Prospective Database. Ann Surg Oncol 2017;24:2319-25.

10. Watzka FM, Fottner C, Miederer M, et al. Surgical therapy of neuroendocrine neoplasm with hepatic metastasis: patient selection and prognosis. Langenbecks Arch Surg 2015;400:349-58.

11. Slotta JE, Schuld J, Distler S, et al. Hepatic resection of non-colorectal and non-neuroendocrine liver metastases - survival benefit for patients with non-gastrointestinal primary cancers - a case-controlled study. Int J Surg 2014;12:163-8.

12. Pavel M, Baudin E, Couvelard A, et al. ENETS Consensus Guidelines for the management of patients with liver and other distant metastases from neuroendocrine neoplasms of foregut, midgut, hindgut, and unknown primary. Neuroendocrinology 2012;95:157-76.

13. Mayo SC, de Jong MC, Bloomston M, et al. Surgery versus intra-arterial therapy for neuroendocrine liver metastasis: a multicenter international analysis. Ann Surg Oncol 2011;18:3657-65.

14. Worhunsky DJ, Krampitz GW, Poullos PD, et al. Pancreatic neuroendocrine tumours: hypoenhancement on arterial phase computed tomography predicts biological aggressiveness. HPB (Oxford) 2014;16:304-11.

15. Poultsides GA, Huang LC, Chen Y, et al. Pancreatic neuroendocrine tumors: radiographic calcifications correlate with grade and metastasis. Ann Surg Oncol 2012;19:2295-303.

16. Ronot M, Cuccioli F, Dioguardi Burgio M, et al. Neuroendocrine liver metastases: Vascular patterns on triple-phase MDCT are indicative of primary tumour location. Eur J Radiol 2017;89:156-62.

17. Kim SH, Kim MA, Shin CI, et al. CT differentiation of poorly-differentiated gastric neuroendocrine tumours from well-differentiated neuroendocrine tumours and gastric adenocarcinomas. Eur Radiol 2015;25:1946-57.

18. Catena L, Bichisao E, Milione M, et al. Neuroendocrine tumors of unknown primary site: gold dust or misdiagnosed neoplasms? Tumori 2011;97:564-7.

19. Marrache F, Vullierme MP, Roy C, et al. Arterial phase enhancement and body mass index are predictors of response to chemoembolisation for liver metastases of endocrine tumours. Br J Cancer 2007;96:49-55.

20. Roche A, Girish BV, de Baere T, et al. Prognostic factors for chemoembolization in liver metastasis from endocrine tumors. Hepatogastroenterology 2004;51:1751-6.

21. Verde F, Fishman EK. Calcified pancreatic and peripancreatic neoplasms: spectrum of pathologies. Abdom Radiol (NY) 2017;42:2686-97.

22. Kim JH, Eun HW, Kim YJ, et al. Pancreatic neuroendocrine tumour (PNET): Staging accuracy of MDCT and its diagnostic performance for the differentiation of PNET with uncommon CT findings from pancreatic adenocarcinoma. Eur Radiol 2016;26:1338-47.

23. Liang $W$, Yang $P$, Huang $R$, et al. A Combined Nomogram Model to Preoperatively Predict Histologic Grade in Pancreatic Neuroendocrine Tumors. Clin Cancer Res 2019;25:584-94.

24. Cieciera M, Kratochwil C, Moltz J, et al. Semi-automatic 3D-volumetry of liver metastases from neuroendocrine tumors to improve combination therapy with $177 \mathrm{Lu}-$ DOTATOC and 90Y-DOTATOC. Diagn Interv Radiol 2016;22:201-6.

25. Filippi L, Scopinaro F, Pelle G, et al. Molecular response assessed by (68)Ga-DOTANOC and survival after (90)Y microsphere therapy in patients with liver metastases from neuroendocrine tumours. Eur J Nucl Med Mol Imaging 2016;43:432-40.

26. Abdulrezzak U, Kurt YK, Kula M, et al. Combined 
imaging with 68Ga-DOTA-TATE and 18F-FDG

$\mathrm{PET} / \mathrm{CT}$ on the basis of volumetric parameters in neuroendocrine tumors. Nucl Med Commun 2016;37:874-81.

27. Ng CS, Chandler AG, Yao JC, et al. Effect of preenhancement set point on computed tomographic perfusion values in normal liver and metastases to the liver from neuroendocrine tumors. J Comput Assist Tomogr 2014;38:526-34.

Cite this article as: Armstrong EA, Beal EW, Shah M, Konda B, Abdel-Misih S, Ejaz A, Dillhoff ME, Pawlik TM, Cloyd JM. Radiographic characteristics of neuroendocrine liver metastases do not predict clinical outcomes following liver resection. Hepatobiliary Surg Nutr 2020;9(1):1-12. doi: 10.21037/ hbsn.2019.06.02
28. Lesurtel M, Nagorney DM, Mazzaferro V, et al. When should a liver resection be performed in patients with liver metastases from neuroendocrine tumours? A systematic review with practice recommendations. HPB (Oxford) 2015;17:17-22.

29. Bertani E, Falconi M, Grana C, et al. Small intestinal neuroendocrine tumors with liver metastases and resection of the primary: Prognostic factors for decision making. Int J Surg 2015;20:58-64. 


\section{Supplementary}

Table S1 Univariate cox regression for overall survival ( $\mathrm{N}=82$ )

\begin{tabular}{|c|c|c|}
\hline \multirow{2}{*}{ Characteristics } & \multicolumn{2}{|c|}{ Overall survival from first liver resection } \\
\hline & Univariate $\mathrm{HR}(95 \% \mathrm{Cl})$ & $P$ value \\
\hline Age (years) & $1.00(0.96,1.04)$ & 0.962 \\
\hline Gender, female & $0.99(0.38,2.59)$ & 0.981 \\
\hline \multicolumn{3}{|l|}{ Health insurance } \\
\hline Private & Ref. & \\
\hline Government & $0.19(0.01,2.47)$ & 0.203 \\
\hline Uninsured & $0.12(0.01,1.49)$ & 0.100 \\
\hline Charlson comorbidity score & $0.81(0.53,1.23)$ & 0.322 \\
\hline Body mass index $\left(\mathrm{kg} / \mathrm{m}^{2}\right)$ & $0.86(0.76,0.96)$ & 0.009 \\
\hline \multicolumn{3}{|l|}{ Primary tumor } \\
\hline \multicolumn{3}{|l|}{ Location of primary } \\
\hline Other & Ref. & \\
\hline Pancreas & $2.58(0.81,8.21)$ & 0.108 \\
\hline Small bowel & $0.94(0.27,3.25)$ & 0.920 \\
\hline Functional & $0.75(0.27,2.12)$ & 0.587 \\
\hline Symptomatic & $0.52(0.10,2.61)$ & 0.425 \\
\hline Tumor size $(\mathrm{cm})$ & $0.89(0.68,1.17)$ & 0.408 \\
\hline Synchronous liver metastasis & $0.54(0.14,2.10)$ & 0.371 \\
\hline \multicolumn{3}{|l|}{ Liver metastasis } \\
\hline \multicolumn{3}{|l|}{ Type of operation } \\
\hline Bisegmentectomy & Ref. & \\
\hline Right hepatectomy & $0.57(0.15,1.89)$ & 0.404 \\
\hline Other & $0.54(0.15,1.89)$ & 0.332 \\
\hline Size of largest metastasis $(\mathrm{cm})$ & $0.99(0.89,1.11)$ & 0.937 \\
\hline Tumor number & $0.66(0.28,1.58)$ & 0.351 \\
\hline \multicolumn{3}{|l|}{ Liver metastasis Ki-67 } \\
\hline$<20 \%$ & Ref. & \\
\hline$\geq 20 \%$ & $2.62(0.99,6.97)$ & 0.053 \\
\hline \multicolumn{3}{|c|}{ Liver metastasis tumor differentiation } \\
\hline Well differentiated & Ref. & \\
\hline Poorly differentiated & $5.67(1.97,16.26)$ & 0.001 \\
\hline
\end{tabular}

